\title{
NANCY FRASERS GERECHTIGKEITSTHEORIE ALS BEITRAG ZUR ANALYSE DER AFFIRMATIVE ACTION POLITIK IN DER BRASILIANISCHEN HOCHSCHULBILDUNG
}

\section{ORIGINAL-ARTIKEL}

GUIMARÃES, Matheus De Oliveira ${ }^{1}$

GUIMARÃES, Matheus De Oliveira. Nancy Frasers Theorie der Gerechtigkeit als Beitrag zur Analyse der Affirmative Action Politik in der brasilianischen Hochschulbildung. Revista Científica Multidisciplinar Núcleo do Conhecimento. 04Jahr, Ed. 11, Band 01, S. 22-38. November 2019. ISSN: 2448-0959, Zugangslink: https://www.nucleodoconhecimento.com.br/bildung-

\section{de/gerechtigkeitstheorie}

\section{ZUSAMMENFASSUNG}

Dieser Artikel sucht die Präsentation der Formulierung der sozialen Gerechtigkeit, die von der amerikanischen politischen Philosophin Nancy Fraser ernannt wurde, durch die sie das soziale Szenario heute aus der binomialen Anerkennungs-Umverteilung interpretiert. Wir werden theoretische Argumente vorlegen, die für die Analyse, Interpretation und Bewertung der brasilianischen Bildungspolitik von interessantem Wert sein können, die direkt mit dem Thema der Affirmative Action-Politik verbunden sind. Von der Darstellung der Frasean-Theorie über soziale Gerechtigkeit und Anerkennung aus versuchen wir, diesen Gedanken panoramisch mit den positiven

1 Doktorand in The Right to Education and Educational Policies aus dem Graduate Program in Education der Päpstlichen Katholischen Universität Minas Gerais (PucMinas). Master-Abschluss in Bildung, verbunden mit der Forschungslinie Diversity, Inklusion und Educational Practices, von der Federal University of Ouro Preto (UFOP). Er hat einen Abschluss in Rechtswissenschaften (UFOP), Philosophie (ICSH) und Pädagogik (FGD) und ist auf Pädagogische Praktiken (UFOP) und Public Policy Management (UFOP) spezialisiert. 
Maßnahmen in Brasilien zu verbinden - insbesondere aus dem Jahrzehnt der 1990. Zu diesem Zweck werden die Hauptargumente von nancy fraser vorgeführt, für die die Gerechtigkeit heute sowohl die Umverteilung von Sozialgütern und Reichtum als auch die anerkennung von Unterschieden durch kultur-valorativo erfordert. Schließlich wird die theoretische Beziehung zwischen Affirmative Actions und frasean Theorie hergestellt.

Schlagworte: Affirmative Actions, Higher Education, Social Justice, Nancy Fraser, race, redistribution-recognition.

\section{EINFÜHRUNG}

Die brasilianische Verfassung von 1988 in inrem Artikel 206, Punkt I, und das brasilianische Gesetz über die Bildungsrichtlinien und -grundlagen von 1996 in Artikel 3, Punkt I, legen fest, dass der Unterricht unter Beachtung des Grundsatzes der gleichen Bedingungen vorgesehen werden muss. Im Einklang mit diesem verfassungsmäßigen Gebot begann die Bildungspolitik mit dem Ziel, die sozialen Ungleichheiten zu verringern, die in der Geschichte Brasiliens vorherrschten - und verschiedene Teile der Gesellschaft zu diskriminieren.

In den letzten zwei Jahrzehnten, in Bezug auf die Behandlung von Rassenthema in Brasilien, haben erhebliche Veränderungen in dem Land, die intensive Veränderungen in der Gesellschaft produziert haben. In diesem Zusammenhang werden positive Maßnahmen einbezogen, die als Initiativen zur Förderung der sozialen Eingliederung verstanden werden, und gleichzeitig werden einzelne Aspekte von Gruppen und Einzelpersonen geschätzt.

Der Zusammenhang zwischen den Diskussionen der Bildungs-, Rechts- und Politikwissenschaften mit Themen im Zusammenhang mit Fragen der Umverteilung und Anerkennung - und insbesondere im Hinblick auf die Rasse - beruht in erheblichem Zusammenhang auf der unbestreitbaren Betonung der Ungleichheiten im sozialen Kontext zu verstehen und zu bewältigen, sowohl in den Machtverhältnissen als auch im Wissen - unausweichlich miteinander verbunden (APPLE; BALL; 
GANDIN, 2013; GILLBORN; LADSON-BILLINGS, 2013; APPLE, 2017). Trotz des Verständnisses vieler von einem vermeintlichepigischen Charakter dessen, was schließlich Diskussionen über Klasse und Kapitalismus betreffen würde, ist es wichtig, die relativ autonome Stärke von Rassenfragen und die Bedeutung der die Besonderheiten des täglichen Lebens derer, die solche Situationen leben (APPLE, 2017).

In diesem Sinne hat die brasilianische öffentliche Politik angesichts der Notwendigkeit, denjenigen, die ihre Chancen auf Bildung vernachlässigt haben, Zugang (und Dauerhaftigkeit) zur Hochschulbildung zu gewähren, eine späte Universalisierung der Hochschulbildung und möglicherweise trotz der beabsichtigten, sie am Ende im Einklang mit dem Verlust der Qualität der Lehre ohne effektiv Demokratisierung des Zugangs. Obwohl sich die Bildungsindikatoren in den letzten zwei Jahrzehnten für alle Unterrichtsbereiche und in allen gesellschaftlichen Gruppen verbessert haben, sind noch immer gravierende Rassenungleichheiten zu beobachten - insbesondere in den frühesten Stadien (HEILBORN; ARA-JO; BARRETO, 2010).

Trotz der Debatte über positive Maßnahmen konzentriert sich die Politik, insbesondere seit dem letzten Jahrzehnt, stark auf das Quotensystem für öffentliche Universitäten, und die Maßnahmen des Staates beinhalten Maßnahmen, die über Quoten hinausgehen - mit Merkmalen und differenzierten Anwendungsbereich.

Obwohl es möglich ist, zu bestätigen, dass in der Regierung Fernando Henrique Cardoso gab es bereits Bundesinitiativen für die schwarze Bevölkerung, die Analyse der Dokumente der Periode zeigt, dass die diskursive Strategie und die Politik dieser Regierung war, anerkennung $z u$ fördern ohne Investitionen in den Umverteilungsaspekt, obwohl Ungleichheit die Hauptbegründung für die Politik der Bewertung der schwarzen Bevölkerung war (LIMA, 2010).

Der Beginn der Regierung lula im Jahr 2003 markiert einen dichten Politikwechsel mit rassischer Perspektive. Wie Lima (2010) feststellt, war das Verhältnis des Staates zur Schwarzen Bewegung bis dahin von Äußerlichkeit, die im Grunde soziale Akteure als Kläger und mit wenig Einfügung in den Regierungsapparat darstellte. In lulas 
Regierung wird diese Beziehung aus der Einbeziehung schwarzer Repräsentanten in ihren Rahmen (die den Forderungen der Bewegung mehr Sichtbarkeit verleihen).

Tatsache ist, dass das Thema der Hochschulbildung sicherlich das Thema ist, das die öffentliche Debatte über affirmative Aktionen mobilisiert - insbesondere im Hinblick auf die Umsetzung von Quotenpolitiken in öffentlichen Hochschulen und Analysen zu Studenten. Quoteninhaber. Wie Lima (2010) feststellt, hatte die ausgeprägte soziale Reaktion auf die Quotenpolitik jedoch nicht die gleiche Reaktion, als die Regierung ein Programm positiver Maßnahmen im privaten System umsetzte - verantwortlich für mehr als $80 \%$ der Einschreibungen in die brasilianische Hochschulbildung. .

Die wichtigsten öffentlichen Politiken des föderalen Bereichs mit rassischem Ausschnitt in der Bildung sind heute sowohl im Bereich der kulturellen Anerkennung als auch im Bereich der Umverteilung organisiert. In Brasilien, zusätzlich zu der berüchtigten Quotenpolitik (oft fälschlicherweise als Synthese von Affirmative Actions verstanden), PROUNI (University For All Program), über die die Bundesregierung Stipendien (integral e für einkommensschwache Studierende, damit sie an privaten Hochschulen studieren können; fies (Studentenfinanzierungsfonds), ein Programm des Bildungsministeriums zur Finanzierung der Ausbildung von Studenten, die an nicht freien Einrichtungen eingeschrieben sind; und das PBP (Permanence Scholarship Program), eine finanzielle Unterstützung (die von der Bundesregierung für einkommensschwache Studierende an öffentlichen Bundesuniversitäten und für Studierende mit Vollstipendien von PROUNI angeboten wird), die darauf abzielt, soziale Ungleichheiten und zur Dauerhaftigkeit und zum Abschluss von Studenten in einer Situation sozioökonomischer Anfälligkeit beitragen.

Von diesen Politiken ist Prouni natürlich die umverteilendste positive Aktion, die in der Geschichte der Hochschulbildung im Land eine größere Wirkung hat. Die 2004 unter Lula durch die vorläufige Maßnahme 213/2004 gegründete Prouni wurde 2005 durch das Gesetz 11.096/2005 institutionalisiert. Dieses Programm zielt darauf ab, einkommensschwache Studierende in Bachelor- und Sequenzstudiengängen an privaten Hochschulen mit einem Teilstipendium zu beauferten. Steuerbefreiungen werden Instituten angeboten, die dem Programm unterliegen. 
Pereira bestätigt die Idee einer gekürzten Demokratisierung des Zugangs zur Hochschulbildung durch positive Maßnahmen und verweist indirekt auf die Ergebnisse von Prouni:

[...] der private Sektor in Brasilie[responde]n für $88 \%$ des Angebots an offenen Stellen und für $71 \%$ der Einschreibungen, was es zu einem der größten in der Welt mach[...]t. Mit Hilfe des privaten Netzwerks deckt das Angebot an offenen Stellen für die Hochschulbildung heute $86 \%$ der Hochschulabsolventen ab. [Contudo][...]nur $10 \%$ der Jugendlichen im Alter von 18 bis 24 Jahren nehmen daran teil (2009, S. 45).

Pereira (2009) fügt hinzu, dass leerstande Stellen in privaten Hel vor der Einführung von Prouni bei 49,5\% lagen - während diese Quote in den staatlichen HeK-Behörden $0,9 \%$ und im Staat $4,7 \%$ betrug.

Zur Politik des Ausbaus der Hochschulbildung informiert Pereira:

Das wirtschaftliche Szenario, das wir erleben, zeigt, dass die Auswirkungen der Demokratisierung im Bildungsbereich nicht zu einer Sozialdemokratisierung führen, sondern dass sie neue und schmerzhaftere Ungleichheiten hervorrufen können. (2009, p. 46).

Und genau als Vorschlag für die Untersuchung dieses komplexen Kontextes wird die Analyse der von Nancy Fraser vorgestellten Theorisierungen (2008, 2003, 2002, 2000 , 1997) aufzeigt. Wären positive Maßnahmen zur Demokratisierung des Zugangs und der Dauerhaftigkeit von Minderheiten in der Hochschulbildung wirksam zur Förderung des sozialen Wandels? Würden sie aus wirtschaftlicher Sicht genauso wirksam sein? Und wie wirken sich Ihre Zielgruppe auf den Bereich der kulturell-valertierenden Anerkennung aus?

In diesem Text sind keine schlüssigen Antworten auf diese Fragen vorgesehen. Es wird jedoch angenommen, dass das Verständnis von frasean Argumenten in diesem Zusammenhang von interessantem Wert für diese Analyse ist.

\section{WIRTSCHAFTLICHE-DISTRIBUTIVE POLITIK $X$ KULTURVALUATION SIVE POLITIK: NANCY FRASER'S THEORIZATIONS AUF DER DILEMMA REDISTRIBUTION-}




\section{RECOGNITION ALS E[2]INE SUBVENTION FÜR DIE INTERPRETATION DER AFFIRMATIVE ACTION POLICIES IN BRAZILIAN HIGHER EDUCATION}

Nancy Fraser (2008, 2003, 2002, 2000, 1997) verweist auf den Kampf um Anerkennung als etwas, das in Schritten zur paradigmatischen Form des politischen Konflikts seit dem Ende des letzten Jahrhunderts wird. Fraser (2008, 2003, 2002, 2000, 1997) zufolge befeuern die Forderungen nach Anerkennung des Unterschieds die Kämpfe von Gruppen, die unter verschiedenen Flaggen mobilisiert wurden Nationalität, ethnische Zugehörigkeit, Rasse, Geschlecht und Sexualität. Folglich geht die Gruppenidentität darüber hinaus, in diesen Konflikten (sogenannte Postsozialisten) das Klasseninteresse selbst als Hauptmittel der politischen Mobilisierung: "Kult[a]urelle Herrschaft verdrängt Ausbeutung als grundlegende Ungerechtigkeit. Und die kulturelle Anerkennung tritt an die Stelle der sozioökonomischen Umverteilung als Mittel gegen Ungerechtigkeit und das Ziel des politischen Kampfes" (FRASER, 2008, S.11, Übersetzung von mir).

Dies ist jedoch nicht alles - da sich die Kämpfe um Anerkennung in einer Welt verschärfter materieller Ungleichheit entwickeln - insbesondere in den südlichen Ländern.

In diesem Zusammenhang wirft Fraser (2008, 2003, 2002, 2000, 1997) in seinen Theorethesen einige Fragen auf, die seine Debatte über die Dilemmata der Justiz skizzieren werden:

Wie also sollten wir die Verfinsterung eines sozialistischen Imaginären sehen, das sich auf Begriffe wie Zinsen, Exploration und Umverteilung konzentriert? Und was sollten wir mit der Entstehung einer neuen politischen Bildsprache tun, die sich auf Vorstellungen von Identität, Differenz, kultureller Herrschaft und Anerkennung konzentriert? Stellt dieses Gähnen einen Verfall des falschen Bewusstseins dar? Oder wäre es stattdessen eine Möglichkeit, die kulturelle Blindheit eines marxistischen Paradigmas zu kompensieren, das durch den Zusammenbruch des Sowjetkommunismus in Verruf gebracht wurde? (FRASER, 2008, S.11, Übersetzung von mir, Autor griffins). 
Kategorisch erklärt Fraser (2008, 2003, 2002), dass keine dieser Positionen angemessen wäre, und charakterisiert sie als "zu umfassend und ohne Nuancen". Anstatt einfach eine einfache Tonne in der Identitätspolitik zu bestätigen oder abzulehnen, sollte man davon ausziehen, dass eine intellektuelle und praktische Herausforderung vor uns liegt: die Entwicklung einer kritischen Theorie der Anerkennung, die die verteidigt nur die Versionen der Kulturpolitik der Differenz, die konsequent mit der Politik der sozialen Gleichstellung kombiniert werden können (FRASER, 2008, S. 11-12).

Fraser (2008) erklärt bei der Formulierung dieses Projekts, dass die Gerechtigkeit heute sowohl Umverteilung als auch Anerkennung erfordert, und schlägt vor, die Beziehung zwischen den beiden zu untersuchen. Dies, so der Autor, würde zum Teil bedeuten, darüber nachzudenken, wie kulturelle Anerkennung und soziale Gleichheit so konzipiert werden können, dass beide sich selbst erhalten - anstatt sich selbst zu vernichten (da es mehrere konkurrierende Vorstellungen zwischen ihnen gibt). Darüber hinaus würde es auch bedeuten, die Mittel zu theoretieren, mit denen wirtschaftliche Benachteiligung und kulturelle Missachtung miteinander verflochten sind und sich gegenseitig unterstützen. In diesem Sinne verlangt die Justiz für den Autor auch, dass die auftretenden politischen Dilemmata geklärt werden, wenn wir versuchen, beide Ungerechtigkeiten gleichzeitig zu bekämpfen (FRASER, 2008, S. 12).

Fraser behauptet, als einer seiner Zwecke:

(...) zwei politische Probleme, die sich jetzt voneinander distanzieren. Nur mit der Integration zwischen Anerkennung und Umverteilung werden wir einen konzeptionellen Rahmen erreichen, der den Anforderungen unserer Zeit entspricht (FRASER, 2008, S.12, Übersetzung von mir).

Fraser (2008, S. 13-18) präsentiert zwei allgemeine Formen des Verständnisses von Ungerechtigkeit.

Die erste wäre diejenige, die sich auf wirtschaftliche Ungerechtigkeit bezieht - die ihrer Meinung nach in der wirtschaftspolitischen Struktur der Gesellschaft (Ausbeutung, wirtschaftliche Marginalisierung und Entbehrung) verwurzelt ist und deren 
Konzeptualisierung ihrer Natur bereits durch von egalitären Theoreten (FRASER, 2008, S. 13-14).

Der zweite Weg, Ungerechtigkeit zu verstehen, ist laut Fraser (2008) kulturelle oder symbolische, sich vertiefende Ungerechtigkeit in den sozialen Mustern der Repräsentation, Interpretation und Kommunikation - deren Beispiele, so der Autor, kulturelle Dominanz umfassen (über die Unterwerfung unter Interpretations- und Kommunikationsmuster, die mit einer anderen Kultur verbunden sind, die nicht mit ihrer eigenen verbunden und/oder feindlich ist); Verschleierung (im Zusammenhang mit der Invisibilisierung durch die Wirkung kommunikativer, interpretatorischer und gegenständischer Praktiken, die von der Kultur selbst akzeptiert werden); und Respektlosigkeit (gekennzeichnet durch Diffamierung und routinemäßige Disqualifikation in stereotypen öffentlichen kulturellen Darstellungen und/oder in den Wechselwirkungen des Alltags) (FRASER, 2008, S. 14).

Trotz der Unterschiede zwischen diesen beiden Formen der Ungerechtigkeit (sozioökonomische und kulturelle), Fraser $(2008,2003,2002,2000)$ stellt jedoch fest, dass beide in den heutigen Gesellschaften deutlich präsent sind. Beide sind in Prozessen und Praktiken verwurzelt, die für einige Gruppen Nachteile im Vergleich zu anderen fördern. In diesem Sinne müssen sowohl die eine als auch die andere Form der Ungerechtigkeit behoben werden - wenn auch auf unterschiedliche Weise.

Für Fraser (2008, 2003, 2002, 2000) wäre das Mittel gegen wirtschaftliche Ungerechtigkeit eine Art politisch-ökonomische Umstrukturierung, die eine Einkommensumverteilung, eine Neuordnung der Arbeitsteilung, demokratische Investitionskontrollen oder Transformation anderer grundlegender Wirtschaftsstrukturen. Der politische Philosoph schreibt dieser Reihe von Heilmitteln für wirtschaftliche Ungerechtigkeit den Oberbegriff Umverteilung zu.

Im Gegensatz dazu, so der Autor, wäre das Mittel gegen kulturelle Ungerechtigkeit eine Art kultureller oder symbolischer Wandel. Dies könnte die Bewertung der missachteten Identitäten (sowie der kulturellen Produkte der diffamierten Gruppen), die positive Wertschätzung der kulturellen Vielfalt und, noch radikaler, eine 
umfassende Transformation der Sozialstandards umfassen. Repräsentation, Interpretation und Kommunikation, um die Bedeutung des Selbst und aller Menschen zu transformieren. Fraser $(2008,2003,2002,2000)$ gibt dieser Reihe von Abhilfemaßnahmen für kulturelle Ungerechtigkeit den Begriff allgemeine Anerkennung.

Für den Autor (FRASER, 2008) sind verteilungsfördernde Ungerechtigkeiten konfrontiert, die keine Umverteilungsmittel haben (deren Logik darin bestünde, homogenisierung und die folgemten Fehlcharakterisierung von Gruppen). Wenn wir uns wiederum mit Kollektivitäten befassen, die sich der idealen Art der verachteten Sexualität nähern, werden beispielsweise negative Diskriminierungsungerechtigkeiten konfrontiert, die Anerkennungsmittel benötigen (deren Logik wiederum die Aufwertung der gruppe aus der Anerkennung ihrer Besonderheiten). Doch wenn es sich von den Enden entfernt, verschwimmen die Dinge und die Differenzierung wird viel komplexer und heikler. Fraser (2008) zufolge gibt es bei der Betrachtung von Kollektivitäten, die sich im Zwischenbereich des konzeptionellen Spektrums befinden, Hybridtypen, die Merkmale der ausgebeuteten Klasse mit Merkmalen verachteter Sexualität vermischen. Diese Kollektivitäten werden, wie der Autor vorhersagt, bivalent genannt. Sie unterscheiden sich als Kollektivitäten sowohl aufgrund der politischen Wirtschaftsstruktur als auch der kulturell-wertigen Struktur der Gesellschaft:

Diese bivalenten (unterdrückten oder untergeordneten) Kollektivitäten leiden unter Ungerechtigkeiten, die gleichzeitig auf die politische Ökonomie und Kultur zurückgehen. Kurz gesagt, wie Fraser informiert, können diese Kollektivitäten unter schlechter sozioökonomischer Verteilung und kultureller Missachtung leiden, so dass keine dieser Ungerechtigkeiten an sich eine indirekte Wirkung des anderen ist - aber sowohl primäre als auch kooriginelle. In diesem Fall würden weder Umverteilungsmedikamente noch solche der Anerkennung allein ausreichen. Fraser stellt kategorisch fest: "Bivalente Kollektivitäten fehlen beides" ([redistribuição e reconhecimento]FRASER, 2008, S. 23, meine Übersetzung).

Je nach Fraser sind Geschlecht und Rasse Paradigmen bivalenter Kollektivitäten. Obwohl sie untereinander ungeteilte Besonderheiten haben, decken sowohl 
wirtschaftliche als auch kulturell-wertige Dimensionen ab: "Geschlecht und Rasse implizieren daher sowohl Umverteilung als auch Anerkennung" (FRASER, 2008, S. 23, Übersetzung von mir).

So, so Fraser (2008, S. 25, meine Übersetzung), "ist Rasse [...]eine bivalente Art der Kollektivität".

Einerseits ähnelt der Kampf gegen rassismus der Klasse, da er ein strukturelles Prinzip der politischen Ökonomie ist. In dieser Hinsicht strukturiert die Rasse die kapitalistische Arbeitsteilung. Schlecht bezahlte, schlecht bezahlte Berufe, langweilige, schmutzige und häusliche Berufe werden überproportional von $S c h{ }^{[3]}$ warzen besetzt, während die am besten bezahlten Berufe mit höherem Status, Angestelltem Kragen, Fachleuten, Techniken und sind meist von Weißen besetzt.

Darüber hinaus strukturiert die Rasse nach Angaben des Autors (2008, 2003, 2002, $2000,1997)$ auch den Zugang zum formellen Arbeitsmarkt, indem sie weite Teile der schwarzen Bevölkerung als Unterklasse (auch für den Betrieb selbst missachtet) und vom System ausgeschlossen Produktiv. Infolgedessen gibt es eine politischökonomische Struktur, die Formen der Ausbeutung, Marginalisierung und Entbehrung hervorbringt, die speziell durch Rasse gekennzeichnet sind: "Diese Struktur stellt Rasse als eine wirtschaftspolitische Differenzierung dar, die mit bestimmten Merkmale" (2008, S. 26, meine Übersetzung).

In dieser Hinsicht, so der Autor (2008), erscheint rassistische Ungerechtigkeit als eine Art Verteilungsungerechtigkeit, die eine Umverteilungsentschädigung erfordert. Daher erfordert Rassengerechtigkeit in sehr ähnlicher Weise wie die Klasse die Transformation der politischen Ökonomie, um diese Rassenbildung zu beseitigen. Und die Logik des Heilmittels ähnelt der Logik, die sich auf die Klasse bezieht: "Es geht darum, Rasse aus dem Geschäft zu machen" (FRASER, 2008, S. 26, meine Übersetzung). Wenn Rasse nichts anderes ist als eine ökonomisch-politische Differenzierung, dann verlangt die Gerechtigkeit, wie Fraser sagt (2008, 2003, 2002, 2000), dass sie als Unterscheidungskriterium abgeschafft wird - in diesem Sinne. 
Doch wie der Autor sagte, wenn es um Gender geht, ist Rasse und das nicht nur wirtschaftspolitisch. Die Rasse hat auch kultur-valorative Dimensionen, die sie in das Universum der Anerkennung einfügen, die auch Elemente umfasst, die der Sexualität ähnlicher sind als mit der Klasse (FRASER, 2008, S. 26).

Wie Fraser (2008) hervorhebt, ist ein grundischer Aspekt des Rassismus der Eurozentrismus, der sich durch die genehmigte Konstruktion von Normen auszeichnet, die die mit Derieverbundenheit verbundenen Eigenschaften begünstigen. Hinzu kommt der kulturelle Rassismus, der wiederum durch die weitverbreitete Disqualifikation der als schwarz, braun oder gelb kodierten Dinge gekennzeichnet ist (S. 26).

Wie im Fall des Geschlechts sind diese Schäden Eine Ungerechtigkeit der Anerkennung, und daher besteht die Logik des Rechtsbehelfs auch darin, einer speziell abgewerteten Gruppe eine positive Anerkennung zu gewähren.

Fraser $(2008,2003,2002,2000,1997)$ weist daher darauf hin, dass Rasse eine bivalente Art der Kollektivität ist, mit einem ökonomisch-politischen und anderen kulturvalorativen Gesicht - beide Gesichter verflechten sich, dialektisch - zumal die rassistische und eurozentrische Kulturelle Normen werden im Staat und in der Wirtschaft institutionalisiert, während die wirtschaftliche Benachteiligung der Schwarzen ihre Stimme einschränkt.

Um die Rassenungerechtigkeit zu kompensieren, werden politische Ökonomie und Kultur bewegt. Aber wie der Autor im Umgang mit dem Geschlecht belegt, ist der bivalente Charakter der Rasse auch die Quelle eines Dilemmas.

Fraser (2008, 2003, 2002, 2000) stellt fest, dass Geschlecht und Rasse daher dilemmatische Formen der Kollektivität sind. Im Gegensatz zur Klasse, die ein Ende des vom Autor präsentierten konzeptionellen Spektrums einnimmt, und Sexualität, die die andere Spitze einnimmt, sind Geschlecht und Rasse zweiwertig, da sie gleichzeitig in die Umverteilungspolitik und in die Politik der Anerkennung. 
Aber wie könnte die Differenzierung gleichzeitig aufgelöst und die Spezifität einer verachteten Kollektivität aufgelöst werden?

Nachdem man das Dilemma der Umverteilungsanerkennung auf scheinbar unlösbare Weise dargestellt und davon ausgegangen hat, dass Umverteilungsmittel für wirtschaftspolitische Ungerechtigkeit immer soziale Gruppen unterscheiden, während Anerkennungsmittel für Kultur-Valorising-Ungerechtigkeit unterstreicht immer die Differenzierung der gesellschaftlichen Gruppe, Fraser (2008, S. 28) schlägt vor, alternative Vorstellungen von Umverteilung - einerseits - und Anerkennung andererseits zu untersuchen.

Mit bejahenden Rechtsmitteln für Ungerechtigkeit versteht Fraser diejenigen, die darauf abzielen, ungleiche Auswirkungen sozialer Vereinbarungen ohne die zugrunde liegende Struktur, die sie hervorruft, zu korrigieren (2008, S. 28).

Durch transformative Heilmittel hingegen umfasst der Autor diejenigen, die sich auf die Korrektur ungleicher Effekte durch Die Umgestaltung der zugrunde liegenden Gerativa-Struktur konzentrieren (2008, S. 28).

In Bezug auf den Autor ist der grundlegende Kontrast zwischen affirmative heilmitteln und transformativen Heilmitteln für Ungerechtigkeit "terminale Effekte vs. Prozesse, die sie produzieren. Es ist keine allmähliche Veränderung vs. apokalyptische Veränderung" (FRASER, 2008, S. 28, meine Übersetzung).

Fraser (2008) erklärt diese Unterscheidung zunächst im Falle von Abhilfemaßnahmen für kulturelle Ungerechtigkeit. Laut der Autorin sind die bejahenden Mittel für solche Ungerechtigkeiten mit dem verbunden, was sie "Mainstream-Multikulturalismus" nennt - eine Art Multikulturalismus, der vorschlägt, Die Missachtung durch eine Aufwertung von Gruppenidentitäten auszugleichen. ungerechtfertigt abgewertet (wobei der Inhalt dieser Identitäten und die innen zugrunde liegenden Gruppendifferenzierungen unberührt bleiben). Im Gegensatz dazu werden transformative Heilmittel mit Dekonstruktion in Verbindung gebracht, die die Missachtung durch die Transformation der zugrunde liegenden kultur-wertativen Struktur kompensiert. Wie Fraser sagt, 
würde die Destabilisierung bestehender Gruppenidentitäten und Differenzierungen nicht nur das Selbstwertgefühl von Mitgliedern von immer wieder missachteten Gruppen erhöhen, sondern auch das Zugehörigkeitsgefühl und Zugehörigkeit aller (S. 28-29).

Für den Autor geht es nicht um die Auflösung aller Unterschiede in einer einzigartigen und universellen menschlichen Identität, sondern um die Aufrechterhaltung eines Feldes multipler Unterschiede, nicht binär, fließend, immer in Bewegung:

Während affirmative Anerkennungsmittel dazu neigen, bestehende Gruppendifferenzierungen zu fördern, neigen transformative Anerkennungsmittel dazu, sie langfristig zu destabilisieren, um Platz für künftige Umgruppierungen zu schaffen (FRASER, 2008, S. 30, meine Übersetzung).

Ähnliche Unterscheidungen gelten für Abhilfemaßnahmen gegen wirtschaftliche Ungerechtigkeit. Wie Fraser sagt $(2008,2003,2002)$, sind positive Abhilfemaßnahmen für diese Art von Ungerechtigkeit historisch mit dem liberalen Wohlfahrtsstaat verbunden, der durch sie eine Entschädigung für die unheilende schlechte Verteilung anstrebt (während den größten Teil der zugrunde liegenden wirtschaftspolitischen Struktur). Transformative Heilmittel dagegen verbinden sich historisch mit dem Sozialismus und versuchen, die unfaire Verteilung durch die Transformation der bestehenden wirtschaftspolitischen Struktur auszugleichen.

Fraser stellt fest, dass ein Ansatz, der darauf abzielt, Verteilungsungerechtigkeiten auszugleichen, am Ende zu Anerkennungsungerechtigkeiten führt (2008, S. 31).

Affirmative Redistribution setzt ein universalistisches Konzept der Anerkennung voraus (gleicher moralischer Wert der Menschen; von Fraser $(2008,2003,2002)$ als formale Verpflichtung zur Anerkennung betitelt). Die Praxis der bejahenden Umverteilung neigt jedoch, wenn sie verlängert wird, dazu, eine sekundäre Dynamik der stigmatisierenden Anerkennung in Gang zu setzen, die ihrem eigenen formalen Bekenntnis zum Universalismus widersprich ${ }^{[4]}$. Der Autor (2008), der diese Logik mit transformativen Heilmitteln für Klassenverteilungsungerechtigkeiten kontrastiert, sagt voraus, dass sie universalistische Sozialprogramme, hohe Steuern, 
makroökonomische Strategien zur Schaffung von Vollbeschäftigung, einen riesigen nicht marktorientierten öffentlichen Sektor, bedeutende öffentliche und/oder kollektive Eigenschaften und demokratische Entscheidungen in Bezug auf grundlegende sozioökonomische Prioritäten kombinieren. Transformative Umverteilungsmittel neigen dazu, klassendifferenzierung enmitieren zu lassen, soziale Ungleichheit zu verringern, ohne stigmatisierte Klassen schutzbedürftiger Menschen zu schaffen, die als Nutznießer besonderer Großzügigkeit angesehen werden. Fraser stellt fest, dass diese Abhilfemaßnahmen dazu neigen, Gegenseitigkeit und Solidarität in Anerkennungsbeziehungen zu fördern, und so in der Lage sind, Entschädigungen für Umverteilungsungerechtigkeiten anzugehen, um auch bestimmte Ungerechtigkeiten der Anerkennung (2008, S.31-33)

Fraser (2008) stellt fest, dass die transformative Umverteilung (im Allgemeinen) wie die positive Umverteilung ein universalistisches Konzept der Anerkennung voraussetzt: gleicher moralischer Wert der Menschen. Im Gegensatz zur positiven Umverteilung neigt die Praxis der transformativen Umverteilung jedoch dazu, diese Konzeption nicht aufzulösen.

Aus dieser Diskussion, nach Nur den typischen Fällen-reine Ideen an beiden Enden des konzeptionellen Spektrums, und kontrastieren die divergierenden Auswirkungen von affirmativen und transformativen Heilmitteln $\mathrm{zu}$ verwurzelten Verteilungsungerechtigkeiten ökonomisch - einerseits - und auf der einen Seite - und auf der anderen Seite - wird das gesehen: 1. Positive Heilmittel fördern in der Regel die Gruppendifferenzierung, während transformative Heilmittel diese Differenzierung tendenziell destabilisieren und verwischen; und 2. Affirmative Umverteilungsmittel können einen Protest der Verachtung hervorrufen, während transformative Umverteilungsmittel dazu beitragen können, einige Formen der Nichtanerkennung auszugleichen. In diesem Sinne schlägt Fraser $(2008,2003,2002)$ einen Weg vor, das Dilemma der Umverteilungserkennung neu zu formulieren.

Zum Abschluss dieser Diskussion stellt Fraser jedoch folgende Frage: 
[...] in Bezug auf Gruppen, die beiden Arten von Ungerechtigkeit unterworben sind, was wäre die Kombination von Abhilfemaßnahmen, die am besten geeignet wären, die gegenseitigen Eingriffe, die bei der Suche nach Einer Umverteilung und Anerkennung der gleichzeitig? (2008, S. 33, meine Übersetzung).

Und das sollte immer noch problematisiert werden.

\section{ABSCHLIEßENDE ÜBERLEGUNGEN: KONTEXTUALISIERUNG DER FRASEANO-DEBATTE ZUR REALITÄT DER HOCHSCHULBILDUNG IN BRASILIEN}

Es wird bestätigt, dass Brasilien, weil es durch zahlreiche Ungleichheiten, von verschiedenen Ordnungen gekennzeichnet ist, als ein paradigmatisches Modell markiert ist, um über die Anwendung der Phrasentheorie nachzudenken. In diesem Sinne werden Frasers Theorisierungen für die Analyse des brasilianischen Bildungsszenarios vorgeschlagen.

Wie Ferreira (2010) behauptet, hat die Moderne bei ihrer Ankunft in Brasilien ein Land mit Marken des Konservatismus und Traditionalismus gefunden - eine Situation, die in gewisser Weise bis heute in verschiedenen sozialen Sektoren bleibt. In Brasilien haben Vorurteile und Rassenausgrenzung auf verschleierte, nicht institutionalisierte und offen anerkannte Weise stattgefunden (und sind bis zu einem gewissen Grad erhalten). Dies gefährdet in vielen Fällen die Prozesse des Handelns der Regierung. Aktionen wie Affirmative Action-Politiken werden immer wieder als Affront gegen die Chancengleichheit angesehen - auch wenn Ungleichheit, Segregation und Vorurteile sichtbar sind (FERREIRA, 2010).

In diesem Sinne lässt sich die Leistung der öffentlichen Hand im Hinblick auf den Abbau sozialer Ungleichheiten aus Frasers Theorisierungen (2008, 2003, 2002, 2000) über Anerkennung und Umverteilung absehen. Es ist von wesentlicher Bedeutung, dass die Umverteilungs- und Anerkennungspolitik einander nicht widerspricht und sich darüber hinaus von gegenseitigem Respekt leiten lässt (APPLE, 2017, S. 32). 
Affirmative Action Policies sind Teil von Fraser (2008, 2003, 2002), als Mittel gegen soziale Ungerechtigkeiten. Wie Ferreira (2012) jedoch voraussagt, bezieht sich ein wichtiger Punkt, der in dieser Diskussion berücksichtigt werden sollte, auf die Tatsache, dass positive Maßnahmen aus fraseaner Sicht keine absoluten Garantien wären, um die Struktur positiv zu verändern. die Ungleichheiten erzeugt. Das liegt daran, dass sie vielleicht von vornherein die Notwendigkeit der Verteilung an historisch beeinträchtigte Gruppen erkennen, aber nicht notwendigerweise die strukturellen Sackgassen lösen, die solche Ungleichheiten hervorrufen. Es wird erwartet, dass diejenigen, die von diesen Politiken profitieren, - in großem Umfang und langfristig ihre Ergebnisse des sozioökonomischen und kulturellen Wachstums auf generationenübergreifende Weise übermitteln könnten, so dass ihre Nachkommen keine Politische mehr brauchen. dieser Art, um auf dem Vormarsch zu bleiben oder ein Einkommen und ein ähnliches Statusniveau zu erreichen, wie es die Bevölkerung in der Vergangenheit in Brasilien erhalten hat.

\section{REFERENZEN}

APPLE, Michael W. A educação pode mudar a sociedade?. Petrópolis: Vozes, 2017.

APPLE, Michael W.; BALL, Stephen J.; GANDIN, Luís Armando. Mapeamento da sociologia da educação: contexto social, poder e conhecimento. In: Sociologia da Educação: análise internacional. Michael W. Apple; Stephen J. Ball; Luís Armando Gandin (orgs.). Porto Alegre: Penso, 2013.

LIMA, Márcia. Ações afirmativas no governo Lula. In: Revista Novos Estudos. 2010.

FERREIRA, Wallace. Justiça e reconhecimento em Nancy Fraser. Teresina: Jus Navigandi, 2012.

FRASER, Nancy. From Redistribution to Recognition? Dilemmas of Justice in a "Postsocialist" Age. In. Adding Insult to Injury. Nancy Fraser debates her critics. Edinburgh: Verso, 2008. 
Justice Interruptus: Critical Reflections on the "Postsocialist" Condition. London: Routledge, 1997.

Redistribuição ou reconhecimento? Classe e status na sociedade contemporânea. In: Interseções - Revista de Estudos Interdisciplinares. UERJ, ano 4, n.1, 2002.

Recognition without Ethics? Theory, Culture \& Society, v. 18, p. 21-42, 2001.

Da redistribuição ao reconhecimento? Dilemas da justiça na era pós-socialista. In: Democracia hoje: novos desafios para a teoria democrática contemporânea. Jessé Souza (org.). Brasília: Editora Universidade de Brasília, 2000.

FRASER, N.; HONNETH, A. Redistribution or recognition?: a political philosophical exchange. London; New York: Verso, 2003.

GILLBORN, David; LADSON-BILLINGS, Gloria. Educação e Teoria Racial Crítica. In: Sociologia da Educação: análise internacional. Michael W. Apple; Stephen J. Ball; Luís Armando Gandin (orgs.). Porto Alegre: Penso, 2013.

HEILBORN, M. L.; ARAÚJO, L.; BARRETO, A. (Orgs). Gestão de Políticas Públicas em Gênero e Raça: GPP-GeR, Módulo I. Rio de Janeiro: CESPE; Brasília: Secretaria de Políticas Públicas para as Mulheres. 2010.

PEREIRA, Elisabete Monteiro de Aguiar. A universidade da modernidade nos tempos atuais. In: Revista Avaliação, v. 14, n. 1, p. 29-52. Campinas, 2009.

ZONINSEIN, Jonas; FERES JÚNIOR, João. Ação Afirmativa e Desenvolvimento. In: FERES JÚNIOR, João \& ZONINSEIN, Jonas (Orgs.). Ação Afirmativa e Universidade: experiências nacionais comparadas. Brasília: Editora Universidade de Brasília, 2006.

2. Toma-se, aqui, como referência principal para a análise que se propõe, a seguinte obra: FRASER, Nancy. From Redistribution to Recognition? Dilemmas of Justice in a "Postsocialist" Age. In. Adding Insult to Injury. Nancy Fraser debates her critics. Edinburgh: Verso, 2008. 
3. "People of color", nos termos de Fraser.

4. Nos termos de Fraser, essa dinâmica secundária (estigmatizante) pode ser entendida como o efeito de reconhecimento prático da redistribuição afirmativa (2008, p. 31-32).

Eingereicht: Oktober 2019.

Genehmigt: November 2019. 